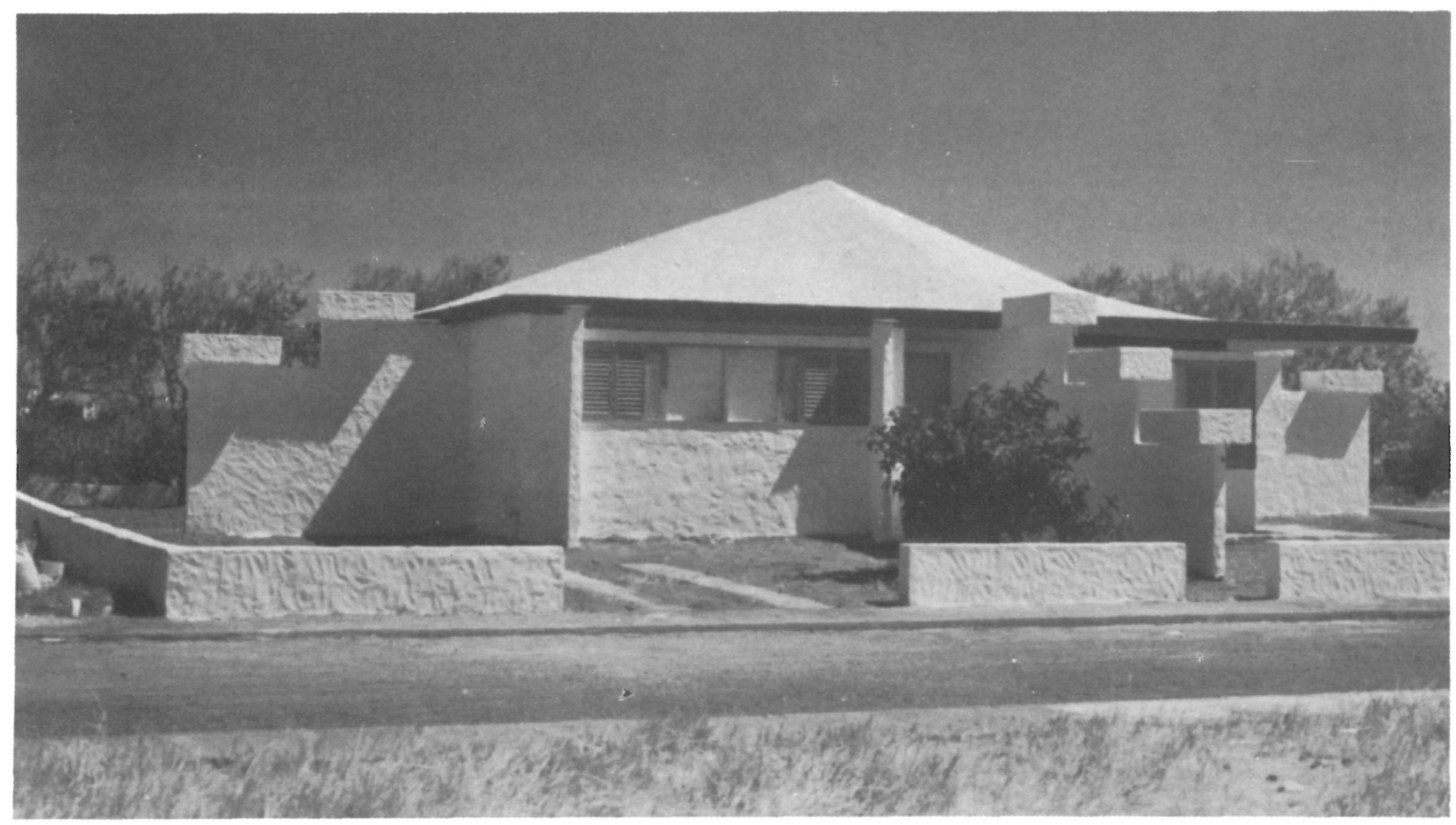

\title{
Casa de campo. Urbanización "Los Almendros», Valsequillo, Gran Canaria
}

En la parte delantera de una pequeña parcela perfectamente rectangular, de 600 metros cuadrados de superficie, localizada en la Urbanización "Los Almendros", se ha levantado esta sencilla casa de campo, cuya planta es un cuadrado de unos $10 \mathrm{~m}$ de lado al que se le han practicado diversos retranqueos para crear las zonas exteriores cubiertas, correspondientes al porche de entrada, terraza y lavadero.

La distribución interior mantiene el mismo sencillo sistema de la planta y se basa en dos muros de carga que, al cortarse ortogonalmente en el centro de la vivienda, establecen la mayor parte de las compartimentaciones necesarias. La organización de las distintas dependencias así obtenidas responde a unos adecuados criterios de zonificación y orientación; de este modo la reservada zona de noche, compuesta por tres grandes dormitorios servidos por un exclusivo y privado cuarto de baño, se encuentra en la parte opuesta a la entrada y orientada hacia levante. Por el contrario, el amplio estar que conforma la zona noble, y que se amplía con la terraza exterior, mira hacia el suroeste y está situado
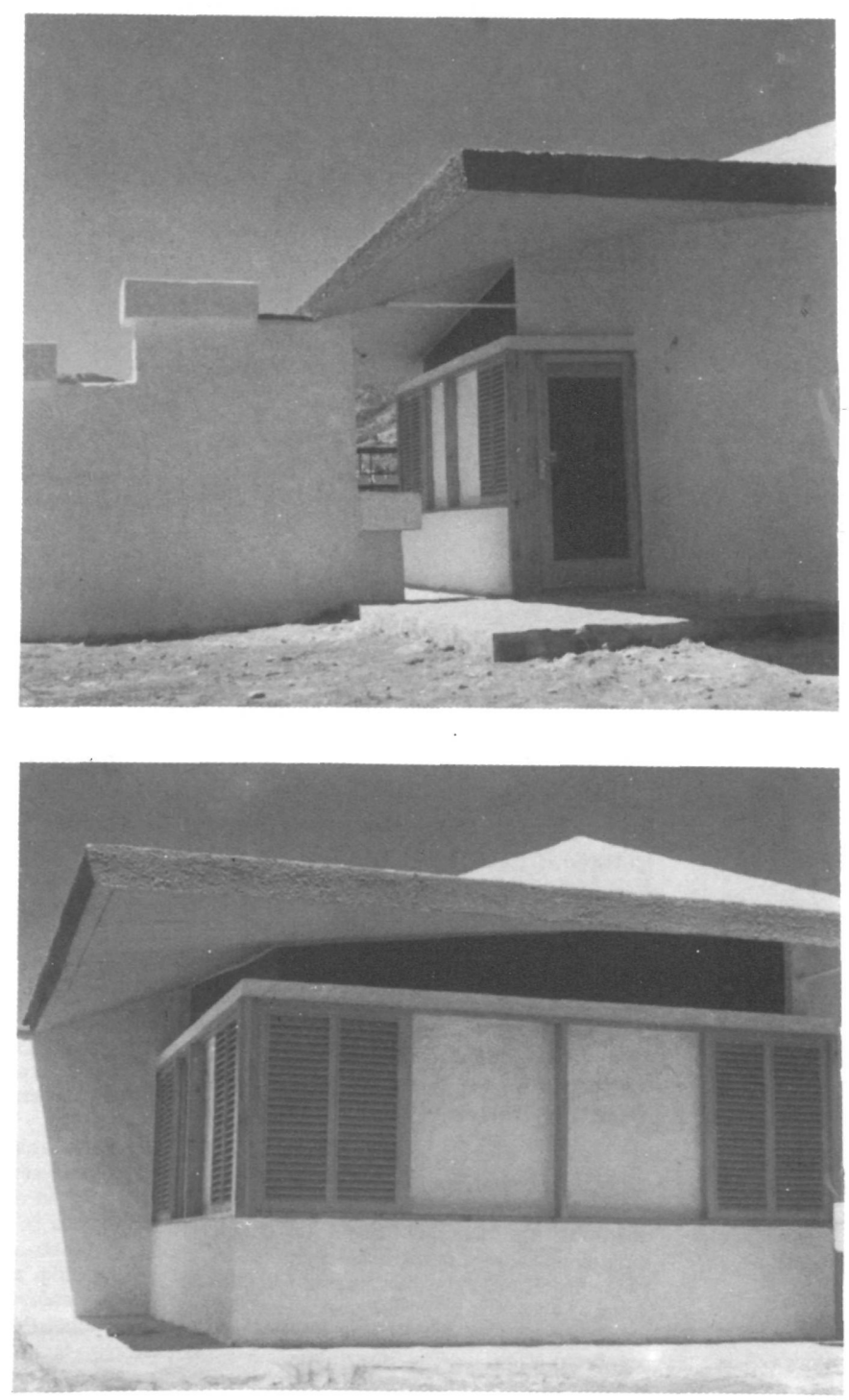


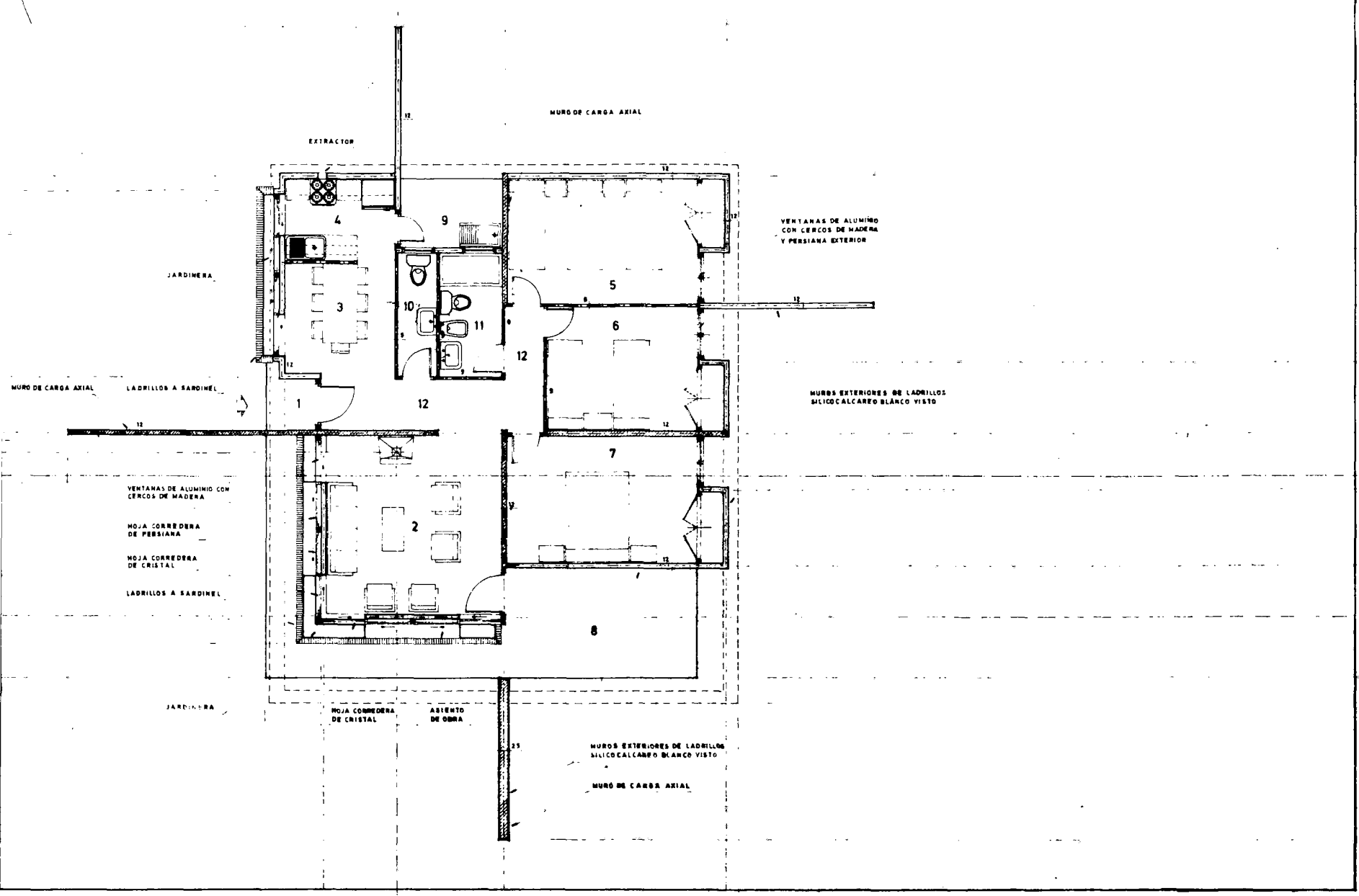

MEDIANThIA

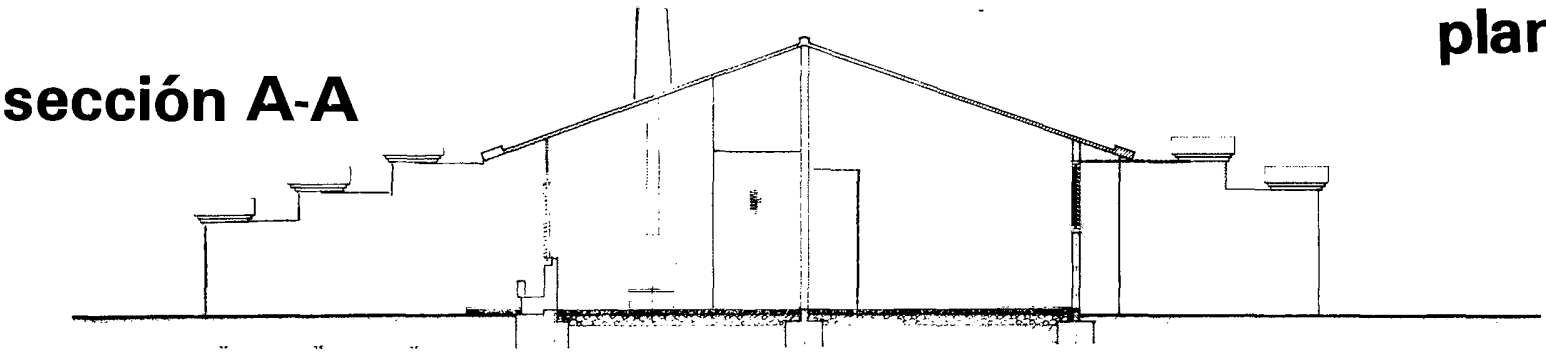

cerca de la entrada, próximo al área de servicio; ésta, a su vez, y también con acceso directo desde el porche de entrada, está orientada hacia el noroeste y formada por una cocina-comedor, un lavadero y un aseo de uso general.

En el exterior destacan los muros de carga encalados que, sobresaliendo perpendicularmente de cada una de las fachadas, y con sus airosas jardineras que los rematan superiormente, constituyen una atractiva característica del aspecto arquitectónico del edificio, proporcionando al mismo tiempo una conveniente zonificación de la parcela.

Pero el elemento más notable y que determinó fuertemente el proceso constructivo es la cubierta, a cuatro aguas, y configurada con superficies regladas - paraboloides hiperbólicos-. Está constituida por membranas delgadas de hormigón armado, de $5 \mathrm{~cm}$ de espesor, protegida superiormente con una capa de $10 \mathrm{~cm}$ de hormigón aligerado e impermeabilizante, y acabada con gravilla de mármol. Su realización fue del siguiente modo: en primer lugar se levantaron únicamente los muros axiles de carga, sobre los que se colocaron unos perfiles y tirantes de hierro previamente soldados, hecho lo cual se completaron los muros axiles en toda su altura; después de montar los cuatro encofrados se armó y hormigonó la cubierta, procediéndose al desencofrado a los 21 días, no sin antes verificar la estabilidad de la misma; por último se levantaron los restantes muros de la casa.

Exteriormente se encalaron todos los paramentos verticales, lo que marca un agradable contraste con la carpintería de madera de puertas y ventanas. 


\section{detalles de cubierta}
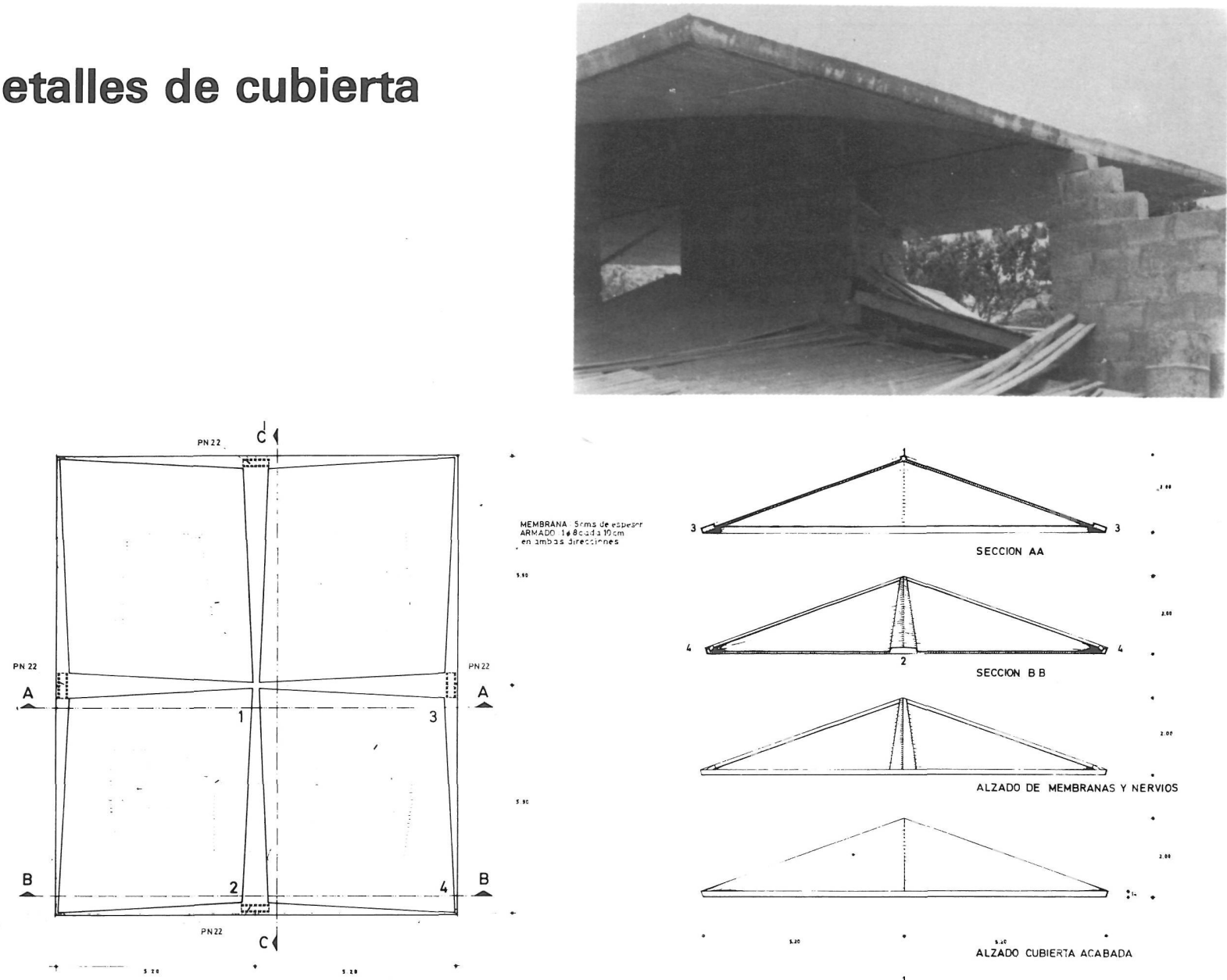

A
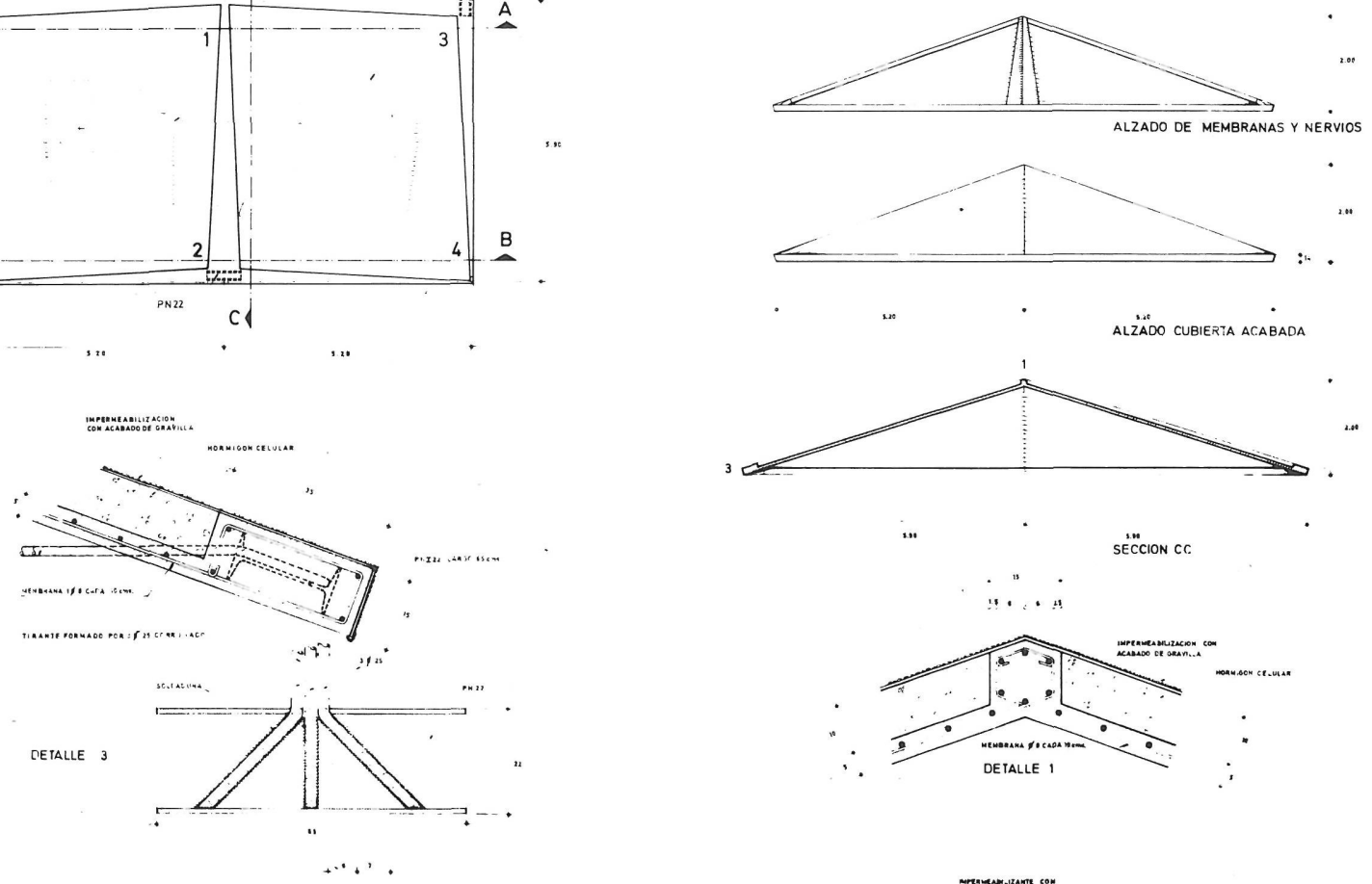

ALZADO CUBIERTA ACABADA
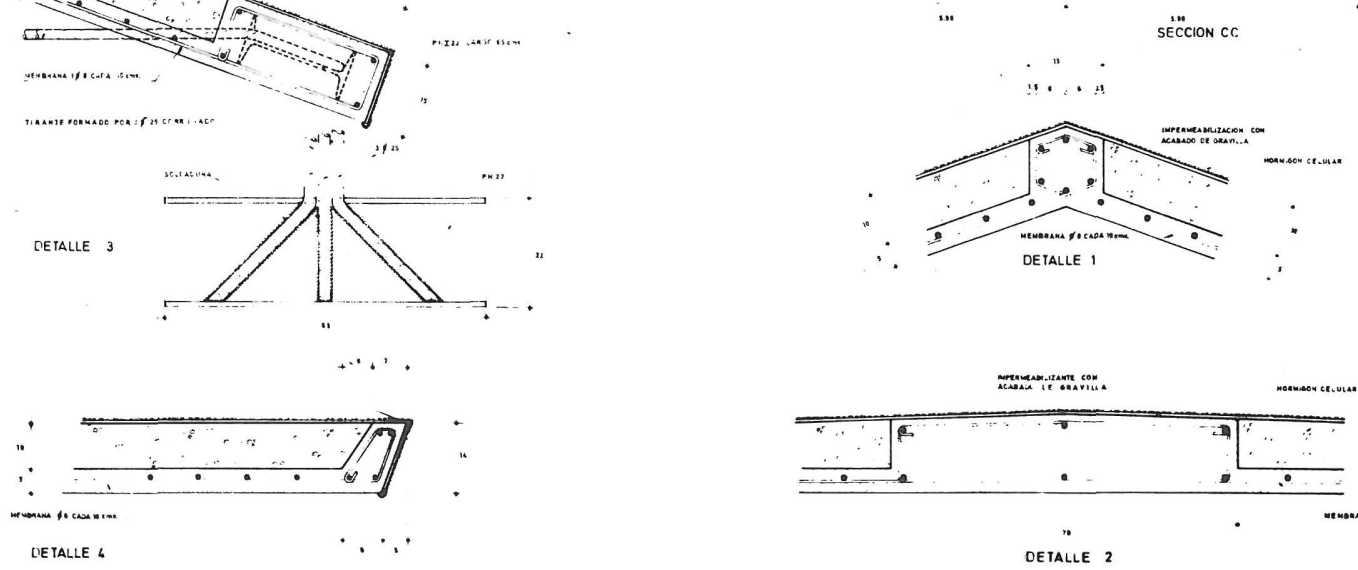

\section{résumé}

MAISON DE CAMPAGNE. LOTISSEMENT "LOS ALMENDROS», VALSEQUILLO, GRANDE-CANARIE

Luis López Díaz, architecte

Cette petite maison de campagne, construite dans le lotissement "Los Almendros", se caractérise par son orientation et par l'organisation, simple mais adéquate des diverses dépendances qui la constituent.

Son aspect extérieur est caractérisé, d'une part, par les murs saillants des façades et les toits résolus à l'aide de paraboloïdes hyperboliques et d'autre part, par le contraste des murs chaulés et des menuiseries en bois.

\section{summary}

COUNTRY HOUSE - «LOS ALMEN DROS» DEVELOPMENT - VALSEQUILLO, GRAN CANARIA

Luis López Díaz, architect

This small country house, built within the "Los Almendros") rural development, is characterised by a simple but adequate layout and distribution of all rooms.

Worth pointing out in the exterior are the bearing walls, projecting out from the façades and the roof designed as hyperbolic paraboloids, and the contrast created between the whitewashed walls and the wooden fenestration.

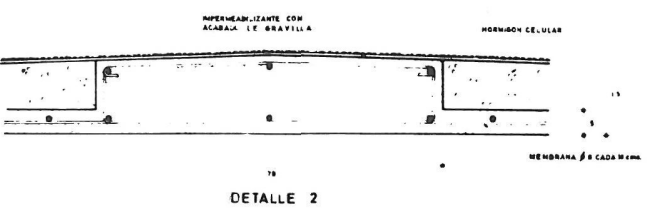

zusammenfassung

LANDHAUS. URBANISATION "LOS ALMENDROS»,

\section{VALSEQUILLO}

\section{Luis López Díaz, Architekt}

Dieses kleine Landhaus, welches in der Urbanisation "Los Almendros» erstellt wurde, zeichnet sich durch eine einfache, jedoch zweckmässige Gliederung und Orientierung der verschiedenen Räume, aus denen es besteht, aus.

Aeusserlich treten einerseits die Stützmauern die aus den Fassaden hervorspringen, sowie die aufgrund von hyperbolischen Paraboloiden ausgeführte trast zwischen den getünchten Mauern und dem Holzwerk hervor. 